\title{
Design of a Compliant and Force Sensing Hand for a Humanoid Robot
}

\author{
Aaron Edsinger-Gonzales \\ Computer Science and Artificial Intelligence Laboratory, Massachusetts Institute of Technology \\ E-mail: edsinger@csail.mit.edu
}

\begin{abstract}
Robot manipulation tasks in unknown and unstructured environments can often be better addressed with hands that are capable of force-sensing and passive compliance. We describe the design of a compact four degree-of-freedom (DOF) hand that exhibits these properties. This hand is being developed for a new humanoid robot platform.

Our hand contains four modular Force Sensing Compliant (FSC) actuators acting on three fingers. One actuator controls the spread between two fingers. Three actuators independently control the top knuckle of each finger. The lower knuckles of the finger are passively coupled to the top knuckle. We place a pair of torsion springs between the motor housing and the hand chassis. By measuring the deflection of these springs, we can determine the acting force of the actuator. The springs also provide compliance in the finger and protect the motor gearbox from high impact shocks.

Our novel actuators, combined with embedded control electronics, allow for a compact and dexterous hand design that is well suited to humanoid manipulation research.
\end{abstract}

\section{Introduction}

Hands for humanoid robots are notoriously difficult to design. Humanoid arms often impose constraints on the size, weight, and packaging of the hand while demanding sufficient dexterity, strength, and speed. Consequently, these hands often lack the ability to sense the force applied by the actuator. They also tend to lack the mechanical robustness necessary for use in unstructured and unknown environments where impacts and collisions are common. Incorporating these features increases the complexity, weight, size, and cost of the hand.

A central pillar of our design approach is that manipulation tasks in unstructured environments require the ability to accurately sense and control the forces exerted by the hand's actuators. Typically, humanoid hands rely on tactile sensors or load cells at the fingertip to gain force knowledge during manipulation. For example, the NASA Robonaut hand[3] utilizes Force Sensing Resistors to sense the pressure at the fingers. The Gifu Hand[2] employs a combination of load cells and tactile sensors. In either case, know-

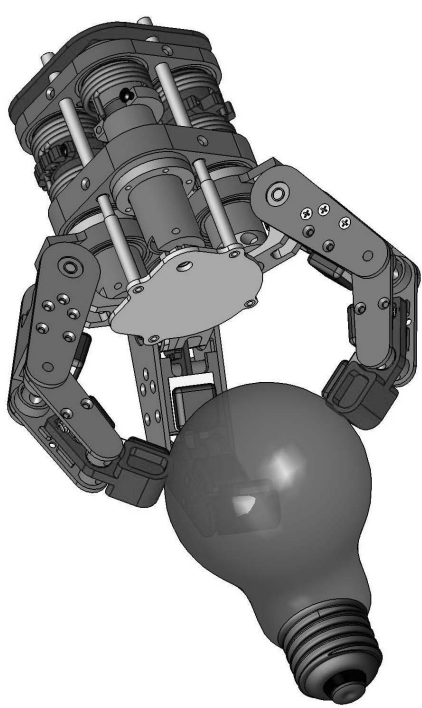

Figure 1: The design for the force sensing and compliant hand.

ing only the fingertip forces may be insufficient when precise knowledge of the manipulating environment is not available, as is often the case in real world environments. Controllers for these hands command finger joint position. This requires accurate knowledge of the position of the manipulated object. Additionally, the fingertip position must be such that the force sensor makes contact with the object for the sensor to be useful.

In contrast, a controller that can command finger joint torque is able to execute a grasp with much less accurate information. The finger need only close with a desired force and the joint position will be determined by the object being grasped. By controlling the grasp in force-space instead of position-space, we can cast the control problem into a form that is intuitive and decomposable[6].

Another emphasis of our design is the incorporation of passive compliance into the hand. Passive compli- 
ance allows for a simple and non-computational means for local, fine-grained adjustments of grasp. The fingertips of our hand are coupled to the preceding link by a spring. A novel aspect of our hand is that the actuators exhibit passive compliance as well. This provides a shock robustness to impacts that would normally damage the actuator.

In the following section we discuss the overall design and specification of the hand along with some of the issues addressed in our particular design. We then analyze, in more detail, the FSC actuators. Finally, we describe the embedded controller developed to interface the hand with a behavior-based humanoid robot research platform.

\section{Overview of the Hand Design}

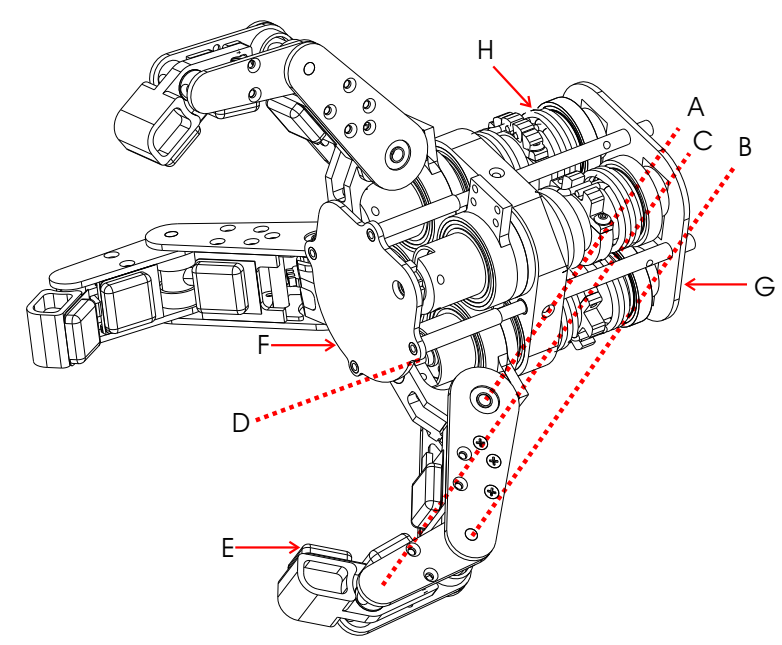

Figure 2: Schematic of hand features: Each of three fingers has three joints $(A, B, C)$. Joint $A$ is driven by a FSC actuator (H) through a cable drive. Joint $B$ is passively coupled to $A$ through a rigid cable drive. Joint $C$ is passively linked by a compression spring to $B$. The spread between two of the fingers (about axis $D)$ is driven by FSC actuator I. The interior surface of each link in a finger has a tactile sensor (E) and the palm has an array of tactile sensors $(F)$. Electronics for motor drive, sensor conditioning, force sensing, and controller interface reside at the rear of the hand (G).

Figure 2 provides an overview of the hand which contains four FSC actuators acting on three fingers. One actuator controls the spread between two fingers. Three actuators independently control the top knuckle of each finger. The lower knuckles of the finger are passively coupled to the top knuckle.

The three fingers are mechanically identical, however two of the fingers can rotate about an axis perpendicular to the palm. These axes of rotation are mechanically coupled through spur gears, constraining the spread between the two fingers to be symmetric.

We place a pair of torsion springs between the motor housing and the hand chassis. By measuring the

\begin{tabular}{|l|l|l|}
\hline Hand & & \\
& Total Weight & $18 \mathrm{oz}$ \\
& Body Dim. & $2.75 \mathrm{x} 2.0 \mathrm{x} 2.0 \mathrm{in}$ \\
& Finger Dim. & $3.66 \mathrm{x} .83 \mathrm{x} 0.7 \mathrm{in}$ \\
& Finger Tip Force & $20 \mathrm{oz}$ \\
& Curl Range & $140 \mathrm{deg}$ \\
& Spread Range & $160 \mathrm{deg}$ \\
\hline Actuator & & \\
& Weight & $3.1 \mathrm{oz}$ \\
& Size & $1.0 \mathrm{x} 1.0 \mathrm{x} 2.75 \mathrm{in}$ \\
& Torque Stall & $77 \mathrm{oz}-\mathrm{in}$ \\
& Torque (continuous) & $28 \mathrm{oz}-\mathrm{in}$ \\
& Speed Max & $3.1 \mathrm{rev} / \mathrm{s}$ \\
\hline Spring & & \\
& Active Coils & 3.25 \\
& Diam. & $0.70 \mathrm{in}$ \\
& Wire Diam. & $.0625 \mathrm{in}$ \\
& Stiffness & $3.85 \mathrm{oz}-\mathrm{in} / \mathrm{deg}$ \\
& Deflection Max & $20 \mathrm{deg}$ \\
\hline
\end{tabular}

Figure 3: The design specification of the hand.

deflection of these springs, we can determine the acting force of the actuator. The springs also provide compliance in the finger and protect the motor gearbox from high impact shocks.

The hand employs a cable-drive tendon system whenever possible. The passively coupled links of the finger are driven by cables. The three actuated knuckles are cable-drive as well. While cable-drive systems can add to the complexity of the design, there are also advantages to such an approach. The WAM Arm[7] demonstrated the ability for cable-drive systems to transmit forces some distance from the actuator with high efficiency and very low backlash. A cable-drive system is a good choice for our design as we are concerned with efficiently transmitting forces back to the actuator so they may be accurately sensed. Gear drives suffer from backlash, mechanical inefficiency, higher weight, and are prone to damage under impact loads. Linkage drives, while significantly less complex, exhibit a non-linear force transfer to the actuator which makes sensing of the force difficult. Direct drive is typically not an option as the packaging constraints of the hand dictate that the motor cannot be axially aligned with the joint.

Figure 3 provides a basic design specification of the hand. The overall size, force capacity, and speed of the hand roughly conforms to that of an adult human hand. We have modeled the kinematic structure after the Barrett Hand[8] which has demonstrated remarkable dexterity and grasping versatility. While only possessing four DOF, the hand can still exhibit a wide variety of grasps, as indicated in Figure 4. 


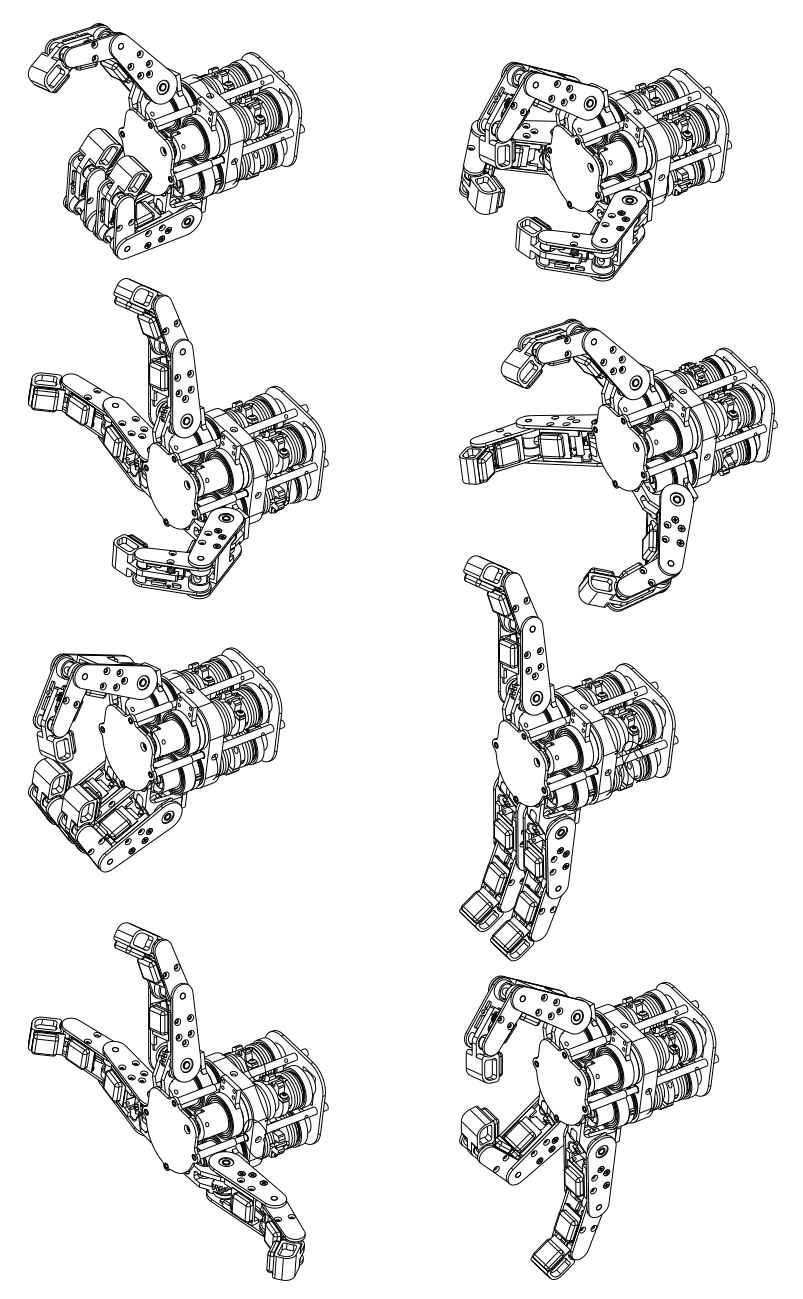

Figure 4: The four DOF in the hand, combined with a high range of motion for each joint, provide a large variety of grasps. The finger span, when open, measures 8.8 inches. Each top knuckle is capable of up to 140 degrees of motion. The spread between the two fingers has a range of 160 degrees.

\section{The Force Sensing Compliant Actu- ator}

The principal component of our design is the Force Sensing Compliant actuator. The FSC actuator provides a very compact method of sensing the force applied by the motor to a load, or conversely, an externally applied force at the finger. This sensor is mechanically simple and consequently inexpensive to manufacture, simple to assemble, and robust to component failure.

Typical robot actuators fasten the motor directly to a chassis (ground). The FSC actuator utilizes two torsion springs which provide a compliant link between the motor body and ground, as pictured in Figure 5. The motor body is suspended between two bearings. When the motor applies a torque to a load, a counteractive deflection of the springs occurs. We can measure this deflection, and by Hooke's Law, we can know the torque applied by the motor.

We sense the spring deflection with a potentiome-

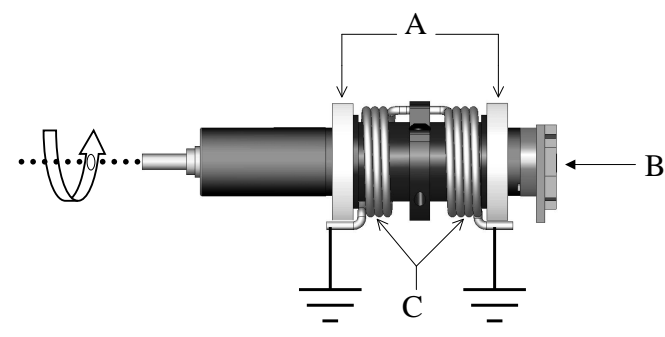

Figure 5: A simplified view of the modular actuator. Two bearings (A) support the motor. The motor is attached to an external frame (ground) through two torsion springs $(C)$. As the motor exerts a torque on a load, a deflection of the springs is created. This deflection is read by the torque sensing potentiometer (B).

ter. The potentiometer shaft is connected to the motor body and the potentiometer body is connected to ground. The potentiometer can be axially aligned with the motor body, allowing for a compact and mechanically simple sensor connection.

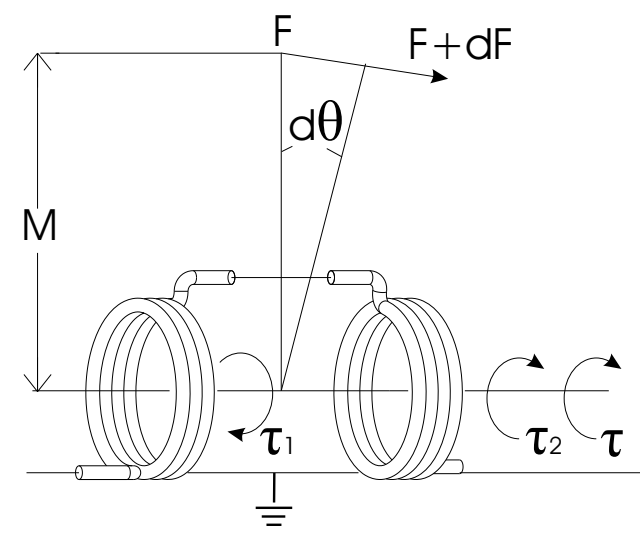

Figure 6: Force diagram for the actuator force sensor. A force perturbation $d F$ causes deflection $d \theta$ of moment arm $M$.

In Figure 6 we illustrate the relationship between the load deflection and sensed force. An externally applied force $F$ creates a torque $\tau$ about moment arm $M$. This torque is counteracted by the springs with spring constant $k$. They are wound in the same direction yet placed face to face, causing them to create torques $\tau_{1}$ and $\tau_{2}$ in opposite directions. If the spring deflection angles are $\theta_{1}$ and $\theta_{2}$, then the torques must balance as:

$$
\tau=\tau_{1}-\tau_{2}=k \theta_{1}-k \theta_{2}
$$

An external force perturbation $d F$ results in torque $\tau^{\prime}$ and a deflection of the torsion springs by $d \theta$. We can sense this perturbation by measuring $d \theta$ : 


$$
\begin{array}{r}
d F=\frac{\tau^{\prime}-\tau}{M} \\
=\frac{\left[k\left(\theta_{1}+d \theta\right)-k\left(\theta_{2}-d \theta\right)\right]-\left[k \theta_{1}-k \theta_{2}\right]}{M} \\
=\frac{2 k d \theta}{M}
\end{array}
$$

We see that the effective spring constant is double that of a single spring.

The selection of the torsion springs is an important part of the FSC actuator design. We had custom torsion springs manufactured to meet our design specifications. The primary considerations are spring stiffness and maximum deflection angle. Designing for spring stiffness is covered in [4]. The spring is chosen such that at the motor stall torque $\tau_{\text {stall }}$ the maximal spring deflection angle $\theta_{\max }$ occurs. This relationship is given by:

$$
\tau_{\text {stall }}=k \theta_{\max }
$$

The value of $\theta_{\max }$ determines the maximum sensor resolution available to the controller. However, it cannot be made arbitrarily large due to the material properties of the spring.

We should also note that both springs need to be precompressed in the resting state of the actuator. Precompressing by $\theta_{\max } / 2$ ensures that at the maximum deflection in one direction, both springs are engaged in counteracting the applied force.

The overall passive compliance exhibited by the actuator is determined by the spring stiffness $k$. If we consider that an externally applied force to the actuator can only be counteracted by the torsion springs, then we see that the mechanical impedance of the system is defined by that of the springs. The low impedance of the springs adversely affects the reaction speed, or bandwidth, of the system. For robot tasks achieved at a roughly human level bandwidth, this adverse affect is not large. However, the low impedance of the system also has advantages. Impact shocks to the finger are dampened by the springs, protecting the motor gearbox from damage. These types of shocks are common in robotic hands and seriously limit the longevity of the hand. The passive compliance also allows the finger to better conform to an object through local, fine-grained adjustments of posture.

The FSC actuator is related to the Series Elastic Actuator (SEA)[5] and exhibits many of the same characteristics. These actuators differ primarily in the placement of the compliant spring element. FSC actuators place a spring between the motor and chassis, while SEA actuators place a spring between the motor output and load. As a result, FSC actuators can provide an unlimited range of motion to the load while the SEA actuator cannot.

\section{The Hand Controller}

The larger research direction of our humanoid robot work takes a behavior based approach to manipula-

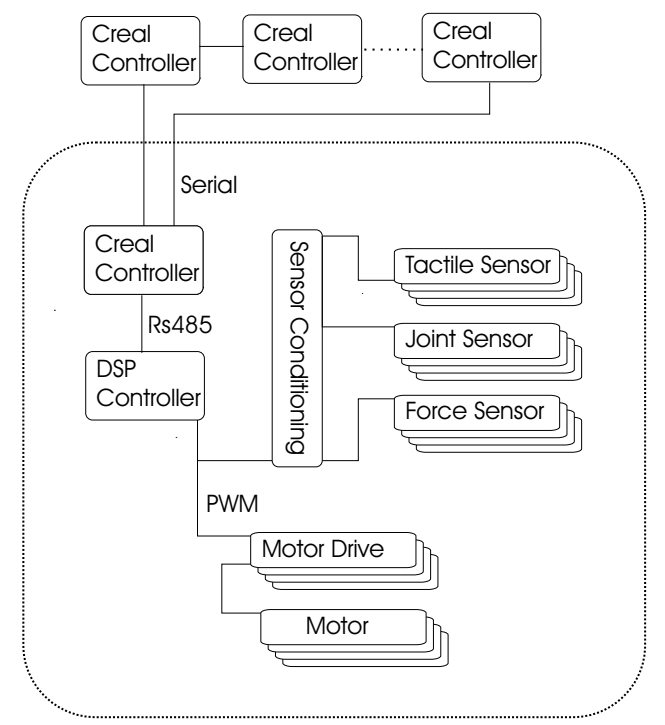

Figure 7: Overview of the hand controller architecture. Signals from sensors embedded in the hand are passed through signal conditioning hardware and read by a $D S P$ control board. The DSP computes the low-level control laws and commands PWM signals to the motor drivers. Higher level control of the hand is handled by a set of behavior based processes which run on a custom Creal controller. The humanoid robot possesses several such embedded controllers which are networked together via a token ring.

tion. Consequently, we have designed embedded, behavior based control electronics for the hand, as outlined in Figure 7. Most of the electronics reside in the hand itself. This limits the number of cables that need be run through the wrist and minimizes robustness issues due to signal noise and cable strain.

The joint angles and actuator torques are sensed by compact potentiometers. Tactile forces are sensed by Force Sensing Resistor (FSR) sensors (See Figure 2 ). These signals are conditioned with a low-pass filter and amplifier and then digitized by a Motorola DSP56F807 controller.

The DSP runs a control loop at $1 k h z$. This control loop performs digitization of each sensor voltage and digitally low-pass filters the value. For each /FSC actuator, it implements a set of control modes using PID controllers. A control mode may implement force control, virtual spring control of joint position [6], or joint velocity control. At the lowest level, all control modes close the loop around the error between the desired actuator torque and the sensed torque. This creates an active spring-like compliance in the fingers which ensures that the finger can robustly withstand unexpected impacts and loads.

A small embedded microprocessor running our behavior based language called Creal[1] manages the behaviors of the hand and the interactions of the hand with the rest of the humanoid system. The Creal controller is located away from the hand and is part of 
a token ring of many such controllers. It communicates to the DSP as a master device on a RS485 bus using a packet protocol run at 100hz. Each packet received by the Creal controller contains the hand's sensor readings. In turn, the Creal controller transmits the desired control mode, the desired setpoint, and the PID gains to the DSP.

We are taking a behavior based approach to the higher level control of the hand. A key component of our approach is the ability to rapidly switch between different control modes. As behaviors are dynamically inhibited and subsumed during the hand's interaction with the environment, the hand can exhibit different control properties. A behavior may employ different combinations of control modes and gains in the execution of its motor action. The details of this approach are beyond the scope of this paper.

\section{Conclusion}

It is our view that manipulation tasks in unknown and unstructured environments can be better addressed with manipulators that are capable of forcesensing and passive compliance. This view has informed our design of a new 4 DOF hand for a humanoid robot. The hand exhibits a low mechanical impedance due to springs placed inline with the actuators and fingers. This low impedance increases the mechanical robustness of the hand, allowing us to safely operate the hand in environments that are not well known. While the hand lacks the dexterity of more anthropomorphic designs, it is capable of a wide variety of grasps. Additionally, we are able to sense and command torques at the actuators. This simplifies the grasping control when tactile or visual features necessary for manipulation are not reliable.

\section{References}

[1] Rodney Brooks. Creature Language. MIT Artificial Intelligence Laboratory, http://www.ai.mit.edu/people/brooks/creal.pdf, September 2003.

[2] Kuzanuao Uchiyama Haruhisa Kawasaki, Tsuneo Komatsu. Dexterous anthropomorphic robot hand with distributed tactile sensor: Gifu hand ii. IEEE Transactions on Mechatronics, 7(3):296303, September 2002.

[3] C. Lovchik and M. Diftler. The robonaut hand: A dexterous robot hand for space. In Proceedings of the IEEE International Conference on Automation and Robotics, volume 2, pages 907-912, Detroit, Michigan, May 1999.

[4] Erik Oberg and Christopher McCauley, editors. Machinery's Handbook. Industrial Press, Inc, 26th edition, April 2000.

[5] Gill A. Pratt and Matthew M. Williamson. Series elastic actuators. In Proceedings of the IEEE/RSJ International Conference on Intelligent Robots and
Systems (IROS-95), volume 1, pages 399-406, Pittsburg, PA, July 1995.

[6] Jerry E. Pratt. Virtual model control of a biped walking robot. Technical Report AITR-1581, MIT Artificial Intelligence Laboratory, 1995.

[7] W. T. Townsend and Salisbury. "Robots and biological systems : towards a new bionics?", chapter Mechanical design for wholearm manipulation. Springer-Verlag, 1993.

[8] William Townsend. The barretthand grasper. Industrial Robot: and International Journal, 27(3):181-188, 2000. 